\title{
Depressão maior e supressão hormonal: resposta com a nortriptilina
}

\section{Major depression and hormone suppression: response with nortriptiline}

\author{
Livia Mitsue Gomes Yukizaki ${ }^{1}$, André Barciela Veras ${ }^{2}$, Flávia Schueler Franco ${ }^{1}$, \\ Luisa Duarte Novo ${ }^{1}$, Arabella Rassi ${ }^{3}$, Antonio Egídio Nardi ${ }^{4}$ \\ 'Graduanda de Medicina da Universidade Federal do Rio de Janeiro (UFRJ). \\ ${ }^{2}$ Mestrando do Programa de Pós-Graduação do Instituto de Psiquiatria da UFRJ. \\ ${ }^{3}$ Aluna do estágio probatório para Mestrado do Programa de Pós-Graduação do Instituto de Psiquiatria da UFRJ. \\ ${ }_{4}^{4}$ Professor adjunto do Instituto de Psiquiatria da Faculdade de Medicina da UFRJ. \\ Recebido: 22/11/2006 - Aceito: 16/03/2007
}

\section{Resumo}

Contexto: A relevância da descrição deste caso clínico é demonstrar a importância do uso de nortriptilina em um caso de depressão maior pós-tratamento para endometriose. Objetivo: Demonstrar as relações entre o tratamento para endometriose e os transtornos psiquiátricos e o resultado terapêutico que obtivemos com o uso da nortriptilina. Métodos: Entrevista psiquiátrica e avaliação clínica psiquiátrica periódica de uma paciente em tratamento no ambulatório do Instituto de Psiquiatria da Universidade Federal do Rio de Janeiro. Resultados: Mulher de 25 anos que, após início de supressão hormonal com goserelina para o tratamento da endometriose, passou a apresentar sintomatologia depressiva e ansiosa proeminente. Sem melhora com o uso de $20 \mathrm{mg} /$ dia de fluoxetina por 8 semanas, foram prescritos $25 \mathrm{mg}$ /dia de nortriptilina com boa resposta clínica em 2 semanas, mantendo a melhora depois de 16 semanas. Conclusão: Apesar de apenas a sertralina ter sua eficácia demonstrada na melhora dos sintomas depressivos associados à supressão ovariana, neste caso a nortriptilina demonstrou-se eficaz. Observamos a necessidade de estudos crescentes na área a fim de avaliar outras opções terapêuticas.

Yukizaki, L.M.G. et al. / Rev. Psiq. Clín. 34(5); 243-245, 2007

Palavras-chave: Endometriose, goserelina, nortriptilina, depressão, ansiedade.

\begin{abstract}
Background: The relevance of this clinical case report is to emphasize the importance of the use of nortryptiline for major depression after endometriosis treatment. Objective: To describe by a case report the relationship between treatment for endometriosis and psychiatric disorders. We will also describe the therapeutic response to nortriptyline. Methods: Psychiatric interview and periodical clinical psychiatric evaluation for the treatment of a patient in the Outpatient Unit of the Institute of Psychiatry of the Federal University of Rio de Janeiro. Results: An outpatient, fifty-five year-old woman with major depression treated in the Psychiatric Institute of Federal University of Rio de Janeiro. She was being treated for endometriosis with goserelin for hormone suppression when she started to complain of depressive and anxiety symptoms. The patient did not improve with $20 \mathrm{mg} /$ day of fluoxetine for 8 weeks. She improved after 2 weeks using $25 \mathrm{mg} /$ day of nortryptiline. The improvement persisted during our 16 weeks follow-up. Conclusion: Although only sertraline has its efficacy demonstrated for depressive symptoms associated with ovarian suppression, in this case nortriptyline was efficient too. We observed the necessity of more studies about this topic in order to better evaluate other therapeutic options.
\end{abstract}

Yukizaki, L.M.G. et al. / Rev. Psiq. Clín. 34(5); 243-245, 2007

Key-words: Endometriosis, goserelin, nortriptyline, depression, anxiety. 


\section{Introdução}

A endometriose é uma doença comum, que acomete $5 \%$ a $10 \%$ das mulheres entre 30 e 45 anos em idade reprodutiva (Shaw, 1992). Caracteriza-se por um crescimento anormal de tecidos semelhantes ao endométrio em volta do útero, ovários e ligamentos (Garner, 1994). Os sintomas mais associados a essa doença são: dor pélvica, dismenorréia, dispareunia, fluxos menstruais irregulares e infertilidade (Shaw, 1992). Existem diversos tratamentos para endometriose, entre eles a terapia com fármacos agonistas do GnRH (hormônio estimulante de gonadotrofinas). O uso dessas substâncias, entre as quais se inclui a goserelina, induz a inibição da síntese e liberação de LH e FSH, hormônios do ciclo menstrual, induzindo um estado de hipoestrogenismo (Damario e Rock, 1994). Esse estado de baixos níveis de estrogênio protege a paciente de um novo episódio de endometriose.

$\mathrm{O}$ uso desse tratamento para endometriose associa-se a um declínio abrupto dos níveis séricos dos hormônios ovarianos, o que pode provocar o aparecimento de sintomas depressivos e ansiosos (Steingold et al., 1987; Warnock e Bundren, 1997). Entre os sintomas encontrados em pacientes em terapia com goserelina, observam-se instabilidade vasomotora, insônia, ressecamento vaginal, fadiga, cefaléia, artralgias e mialgias (Damario e Rock, 1994). Distúrbios psiquiátricos, como a depressão maior, também foram descritos entre mulheres em tratamento com goserelina acompanhadas por 6 meses. Outros estudos também demonstraram essa relação, como o de Warnock et al. (2000), que avaliaram 411 mulheres em tratamento com goserelina e observaram que 54\% apresentaram depressão como efeito adverso, $60 \%$, labilidade emocional e $61 \%$, libido debilitada. Steingold $e t$ al. (1987) observaram transtornos depressivos em $12 \mathrm{de}$ 16 mulheres em tratamento com agonista GnRH. Três das 12 mulheres também apresentaram transtornos de ansiedade/irritabilidade. Warnock et al. (1998), em outro estudo, observaram sintomas depressivos significativos em 16 de 20 mulheres tratadas com goserelina por 24 semanas.

O objetivo deste artigo é exemplificar com um caso clínico as relações entre o tratamento para endometriose e os transtornos psiquiátricos e o resultado terapêutico que obtivemos com o uso da nortriptilina.

\section{Relato de caso}

Paciente do sexo feminino, 25 anos, branca, natural do Rio de Janeiro, solteira e estudante universitária.

Após episódio de dor abdominal súbita e intensa, foi atendida em uma emergência clínica, sendo diagnosticada endometriose. Foi posteriormente submetida à cirurgia videolaparoscópica, quando se retirou apenas o foco de endometriose situado em um dos ovários. Após a cirurgia, iniciou o uso do agonista do GnRH, goserelina $(3,6 \mathrm{mg}$ a cada 28 dias via $\mathrm{SC})$. Obteve supressão da menstruação no segundo ciclo após o início da medicação e não apresentou mais nenhuma queixa dolorosa abdominal.

Três semanas após o início desse tratamento, passou a apresentar tristeza diária, desânimo, anedonia e intensa ansiedade. Observavam-se, ainda, perda do apetite, com relato de perda ponderal não quantificada, insônia e sintomas somáticos ansiosos, como cefaléia, "desconforto no peito” e dores musculares. Não apresentava história pessoal de transtorno psiquiátrico prévio e sua mãe realizava tratamento para "depressão".

O ginecologista responsável manteve o uso de goserelina e recomendou que a paciente procurasse atendimento de um psiquiatra. Efetuada a recomendação, recebeu o diagnóstico de depressão moderada. Iniciou-se o uso de $20 \mathrm{mg} /$ dia de fluoxetina e $1 \mathrm{mg} / \mathrm{dia}$ de alprazolam. Após 8 semanas, apesar do uso correto das medicações prescritas, não obteve melhora dos sintomas. Evoluiu com agravamento do retardo psicomotor, chegando a ficar restrita ao leito por aproximadamente 1 semana. Nesse período, interrompeu completamente suas atividades acadêmicas e sociais.

Motivada pela mãe, procurou atendimento de outro psiquiatra, que, concordando com o diagnóstico do primeiro médico, prescreveu nortriptilina ( $25 \mathrm{mg} / \mathrm{dia})$, substituindo abruptamente o alprazolam e a fluoxetina. Após 2 semanas de tratamento, apresentou melhora drástica dos sintomas, sentindo-se mais animada e retomando suas atividades acadêmicas e sociais adequadamente. Obteve normalização do apetite e adequação do sono, além de alegrar-se com facilidade durante a consulta. Apresentava como queixa residual um leve "desconforto no peito". Após 16 semanas, mantinha melhora descrita e, após 20 semanas do início do uso da nortriptilina, não apresentava mais nenhuma sintomatologia residual.

\section{Discussão}

Diversas citações na literatura relacionam a queda hormonal abrupta com o florescimento de episódios depressivos (Stewarte Boydell, 1993). Tal relação pode estar presente entre mulheres que atingem a menopausa, entre pacientes ooforectomizadas ou em uso de agonistas do GnRH (Stewart e Boydell, 1993; Warnock et $a l ., 2000)$. Essa relação também pode estar mais intensa em mulheres apresentando dor pélvica (86\%) comparativamente a mulheres sem dor (38\%) (Lorençatto et al., 2005). Entretanto, estudos similares demonstraram essa mesma relação em proporções diferentes: $23,5 \%$ de mulheres com queixas de dor pélvica apresentaram depressão, enquanto 13\% do grupo sem dor apresentaram depressão (Waller e Shaw, 1995). Além de comuns, as queixas depressivas e ansiosas que surgem durante o tratamento de supressão hormonal podem comprometer a adesão da paciente. Objetivando uma melhor resposta para o tratamento da endometriose, essas queixas devem ser combatidas de forma eficiente. 
$\mathrm{O}$ uso dos antidepressivos parece ser a alternativa mais óbvia, mas há poucos estudos de eficácia nessa área. Apenas a sertralina teve sua eficácia demonstrada na melhora dos sintomas depressivos associados à supressão ovariana (Warnock et al., 2000; 1998). Poucos sintomas depressivos foram encontrados em pacientes em tratamento com sertralina durante terapia com agonista GnRH em comparação a pacientes somente em terapia com agonista GnRH (Warnock et al., 1998). Os mesmos autores, em um estudo duplo-cego que contou com 33 pacientes, observaram que a sertralina foi superior ao placebo no tratamento de pacientes com sintomas depressivos associados à supressão hormonal ovariana durante terapia agonista GnRH (Warnock et $a l ., 2000)$. Não encontramos na literatura nenhum relato com o uso da nortriptilina.

No caso apresentado, a nortriptilina mostrou-se eficaz para o tratamento da depressão concomitante com o uso de um supressor hormonal. A paciente atingiu recuperação total em doses baixas e em um curto período de uso (2 semanas). A fluoxetina, assim como a sertralina, tem como principal ação o aumento da serotonina na fenda sináptica, mediante inibição da recaptação dessa substância. Pudemos observar que o uso da fluoxetina, durante o período mencionado, demonstrou ausência de resposta e, ainda, piora relativa do quadro depressivo apresentado pela paciente. Portanto, optamos pelo uso da nortriptilina, que, além desse efeito, também aumenta a disponibilidade de noradrenalina, fazendo um paralelo à ação estrogênica, que age aumentando a taxa de degradação da MAO, enzima responsável por degradar a noradrenalina (Sherwin, 1996). Especulamos que, neste caso, este pode ter sido um fator determinante para a pronunciada melhora da paciente, uma vez que esta já havia se apresentado não responsiva à exposição predominantemente serotoninérgica.
Além de bem tolerada, especulamos que a nortriptilina seja tão eficaz quanto os demais antidepressivos para o tratamento da depressão moderada. No caso relatado, mostrou-se mais eficaz que a fluoxetina para o episódio depressivo desencadeado pela supressão hormonal. A nortriptilina pode ser uma boa opção terapêutica também para o tratamento da depressão associada ao tratamento da endometriose pelo seu perfil de boa tolerância, segurança e baixo custo, devendo ser mais bem estudada para a condição particular descrita.

\section{Referências}

Damario, M.A.; Rock, J.A. - Goserelin (Zoladex) versus damazol for endometriosis: The North American Experience. Br J Obstet Gynaecol 101 (suppl 10): 13-18, 1994.

Garner, C. - Uses of GnRH agonists. J. Obstet Gynecol Neonatal Nurs 23: 263-270, 1994.

Lorençatto, C.; Petta, C.A.; Navarro, M.J.; Bahamondes, L.; Matos, A. Depression in women with endometriosis with and without chronic pelvic pain. Acta Obstet Gynecol Scand 00: 1-5, 2005.

Shaw, R.W. - An open randomized comparative study of the effect of goserelin depot and danazol in the tratament of endometriosis. Zoladex Endometriosis Study Team. Fertil Steril 58: 265-272, 1992.

Sherwin, B.B. - Hormones, mood, and cognitive functioning in postmenopausal women. Obstetrics and Gynecology 87(2) (supplement): 1996.

Steingold, K.A; Cedars, M.; Lu, J.K.; Randle, D.; Judd, H.L.; Meldrum, D.R. Treatment of endometriosis with a long-acting gonadotropin-releasing hormone agonist. Obstet Gynaecol 69: 403-411, 1987.

Stewart, D.E.; Boydell, K.M. - Psychologic distress during menopause: associations across the reproductive life cycle. Int J Psychiatry Med 23: 157-162, 1993.

Waller, K.G.; Shaw, R.W. - Endometriosis, pelvic pain and psychological functioning. Fertil Steril 63: 796-800,1995.

Warnock, J.K.; Bundren, J.C. - Anxiety and mood disorders associated with gonadotropin-releasing hormone therapy. Psychopharmacol Bull 33: 311-316, 1997.

Warnock, J.K.; Bundren, J.C.; Morris, D.W. - Sertraline in the treatment of depression associated with gonadotropin-releasing hormone agonist terapy. Biol Psych 43: 464-465, 1998.

Warnock, J.K.; Bundren, J.C.; Morris, D.W. - Depressive mood symptoms associated with ovarian suppression. Fertility and Sterility 74(5): 984-986, 2000. 